\title{
Quantification of bone strength by intraoperative torque measurement: a technical note
}

\author{
Norbert Suhm • Markus Haenni · Ronald Schwyn • \\ Michael Hirschmann · Andreas Marc Müller
}

Received: 22 October 2007 / Published online: 23 February 2008

(C) Springer-Verlag 2008

\begin{abstract}
Introduction Bone strength describes the resistance of bone against mechanical failure. Bone strength depends on both the amount of bone and the bone's quality, and the bone strength may be looked upon as a relevant parameter to judge an osteosynthesis' stability. Information about bone strength was barely available intraoperatively in the past. The previous work of our group reported on development and laboratory evaluation of mechanical torque measurement as a method for the intraoperative quantification of bone strength. With the clinical series presented here we intend to verify that the im gesamten Text DensiProbe ${ }^{\mathrm{TM}}$ instrumentation for intraoperative torque measurement and the related measurement method are eligible for intraoperative use based on the following criteria: application of the method may not create complications, the measurement can be performed by the surgeon himself and may only cause a limited increase in the procedure time.

Patients and methods From December 2006 until May 2007 ten patients with a pertrochanteric femoral fracture or a lateral femoral neck fracture eligible for stabilization with

\author{
N. Suhm $(\bowtie)$ \\ Behandlungszentrum Bewegungsapparat, \\ Universitätsspital Basel, Spitalstrasse 21, 4031 Basel, Switzerland \\ e-mail: nsuhm@web.de \\ M. Haenni · R. Schwyn \\ AO Development Institute, Clavadelerstrasse 8, \\ 7270 Davos Platz, Switzerland \\ M. Hirschmann \\ Department of Orthopedic Surgery, \\ Bruderholzspital, Basel, Switzerland \\ A. M. Müller \\ Department of Orthopedic Surgery, \\ University Hospital, Basel, Switzerland
}

DHS $^{\circledR}$ were included in the study after having received informed consent. Any medication and comorbidity that might have influenced bone quality or bone mineral density (BMD) in these patients was documented. Bone strength was intraoperatively measured with DensiProbe ${ }^{\circledR}$. Complications that were obviously related with torque measurement were documented as well as any deviation from the suggested procedure; 6 and 12 weeks postoperative followup included clinical and radiological examination. The time required for torque measurement, the overall operating time and the number of persons present in the operating room were protocolled. BMD values of the contralateral femoral neck were postoperatively assessed by dual energy X-ray absorptiometry (DEXA) and compared to intraoperative peak torque values measured by DensiProbe ${ }^{\circledR}$.

Results No major complication was observed during intraoperative application of DensiProbe ${ }^{\circledR}$ by trained surgeons. The unintended extraction of the guide wire together with the torque measurement probe was reported only once and is looked upon as a minor complication. Fracture healing was uneventful in all patients. The mean time for torque measurement was $2.35 \pm 0.9 \mathrm{~min}$ accounting for $2.2 \pm 1.1 \%$ of total surgery time. The presence of an additional person was not required to perform torque measurement but to protocol the data. There was a tendency towards correlation between BMD values of the femoral neck and intraoperative peak torque values.

Discussion The data presented clearly indicate that the DensiProbe ${ }^{\circledR}$ instrumentation and measurement principle are eligible for routine intraoperative use by trained surgeons. Interpretation of possible correlations between BMD values measured by means of DEXA and the Peak Torque values assessed by DensiProbe ${ }^{\circledR}$ has to be considered very carefully, because BMD and Peak Torque analyse bone at a different scale. Only within the framework of a multicenter 
study it will be possible to include a sufficient number of patients for calculation of the methods' predictive value towards implant failure and to verify acceptance of the method by the surgeons.

Keywords Bone strength · Proximal femoral fracture · Bone mineral density · Bone densitometry

\section{Introduction}

Proper use of implants and augmentation techniques [1-12] dedicated for fracture fixation in osteoporotic bone follows previous estimation of an osteosynthesis' stability. Several parameters influence the overall osteosynthesis' stability, such as fracture type, quality of fracture reduction [13], implant position [14, 15] and bone strength [16]. All parameters, except for the bone strength, can be rated preoperatively or intraoperatively by the surgeon.

Bone strength, however, depends on the structural and material properties of bone $[16,17]$. In the past there was no measurement principle or instrumentation available in the market for the intraoperative quantification of bone strength. Instead, bone strength is indirectly estimated by bone mineral density (BMD) using dual-energy X-ray absorptiometry (DEXA). Since DEXA-measured BMD accounts for only $60-70 \%$ of the variation in bone strength, some important factors are not captured by DEXA. Moreover, DEXA measurement is not routinely available preoperatively or intraoperatively [18] but can clearly be performed "noninvasively". DEXA measurements might therefore help to estimate the fracture risk of a patient, to put the diagnosis of osteoporosis and to indicate or to monitor an antiresorptive drug therapy of osteoporosis.

On the other hand the assessment of intrinsic mechanical quality of bony tissue should provide a better estimation of bone strength [17]. This option might come into play in patients who have suffered from a fracture and where the surgeon plans to perform osteosynthesis. While performing surgery, the surgeon gets direct access to the bone. Previous work from our group [19] describes development and laboratory evaluation of a new method for intraoperative measurement of bone strength. The torque to breakaway of trabecular bone is measured with the DensiProbe ${ }^{\circledR}$ instrumentation. This should provide immediate and more accurate information about bone strength than the postoperative DEXA scan could. The dimensions and the application scheme of DensiProbe ${ }^{\circledR}$ are adapted for the use with the standard DHS ${ }^{\circledR}$ implant.

\section{Requirements}

Intraoperative application of DensiProbe ${ }^{\circledR}$ instrumentation requires perfect compliance with the hygienic standards in the operating theatre. Furthermore there should be minimal potential for complications due to the intraoperative measurement of bone strength. The surgeon should be able to perform the measurement independently on his own, and the extra time needed for the measurement should be minimal when compared to the overall length of the procedure.

With the pilot series presented here we intended to verify the following hypothesis:

- The DensiProbe ${ }^{\circledR}$ instrumentation is eligible for intraoperative use based on the criteria mentioned above.

Within our stepwise approach to introduce the intraoperative measurement of bone strength into clinical routine, this would mean clarification of the method for evaluation within a multicenter trial.

\section{Materials and methods}

\section{Enrollment of patients}

Following protocol approval by the local ethical committee, patients with proximal femoral fractures having given their informed consent were enrolled in the study from December 2006 until May 2007. The inclusion criteria were defined as the presence of either a stable or unstable pertrochanteric femoral fracture (classified according to $\mathrm{AO}$ as 31A1-A3) [20] or a lateral femoral neck fracture (classified according to Garden as type 1-4) [21] eligible for stabilization with DHS ${ }^{\circledR}$. The lower age limit for inclusion was 35 , in order to assure a wide spectrum of bone qualities was measured during the study. Exclusion criteria were preexisting local infections of the affected hip and pathologic fractures due to primary malignancies or metastatic lesions in the proximal femur. Upon inclusion in the study, all patients were screened for conditions potentially impairing bone strength such as previous surgery in the proximal femur and the presence of major and minor risk factors for osteoporosis (i.e., personal or familiar history of fragility fractures, presence of osteoporotic vertebral fractures, treatment with steroids and anticonvulsants, malabsorption syndrome, physical inactivity, cigarette smoking, low body weight and advanced age). Moreover intake of basic osteoporosis prophylaxis (calcium plus vitamin D) or specific antiosteoporotic therapy (bisphosphonates, SERM, parathormon) was recorded.

\section{Mechanical torque measurement}

Bone strength was measured according to our recently described measurement principle [19], which is fully adapted to stabilization of proximal femoral fracture using the DHS ${ }^{\circledR}$ implant. Fracture reduction and exposure were 
done according to the guidelines for DHS ${ }^{\circledR}$ insertion. Under fluoroscopic control, a K-wire for subsequent guided insertion of the DHS ${ }^{\circledR}$ screw was drilled in the subchondral bone of the femoral head reaching a central-to-inferior position in the AP view and a central-to-posterior position in the axial view. The length of this guide was measured. A second K-wire was inserted in parallel but cranially to the first one in order to prevent rotation of the head-neck fragment during subsequent torque measurement and insertion of the DHS $^{\circledR}$ screw (Fig. 1a, b). Using the $8.0 \mathrm{~mm}$ cannulated spiral drill guided by the lower K-wire, the lateral cortex of the femur was opened. As an adaptation to the standard technique, the spiral drill was inserted to a depth $45 \mathrm{~mm}$ less than the subchondral bone in order to avoid removal of trabecular bone at the site of the following measurement procedure (Fig. 2a, b). After removal of the spiral drill, a custom-made cannulated mechanical probe was inserted over the pre-positioned guide wire into the femoral head. Measurement of bone strength happened at the tip of the rod-like mechanical probe. The tip consists of a wing blade with $6.5 \mathrm{~mm}$ outer diameter and $20.0 \mathrm{~mm}$ blade length (Fig. 3). To position this wing blade at the intended site of the DHS ${ }^{\circledR}$ screw tip at a depth $15 \mathrm{~mm}$ less than the guide wire length, a few gentle hammer blows were applied (Fig. 4a). A scale at the tail of the probe verified the correct insertion depth of the probe (Fig. 4b). The final position of the measurement probe was documented by fluoroscopy (Fig. 4c). Then, a handhold with an intrinsic calibrated digital torque meter (AO Development, Davos, Switzerland) was locked at the lateral end of the probe, which was subsequently rotated around its longitudinal axis (Fig. 5a). The peak torque until complete breakaway of the cancellous bone between the wings of the mechanical probe was recorded by the digital torque meter located in the handhold (Fig. 5b). The digital meter registered the torque values in the range from 1 to $8 \mathrm{Nm}$. A minimal torque of $1 \mathrm{Nm}$ has to be applied in order to trigger the measurement process. This lower limit was chosen in order to prevent the unintended activation of torque recording. If the torque necessary to break away the cancellous bone exceeded $8 \mathrm{Nm}$, the system would unlock allowing free rotation to prevent rotation of the head and neck fragment. The data were processed with MATLAB Software (The MathWorks, Massachusetts, USA). The surgeon performing the measurement procedure was blinded to the result. Then the probe was removed and the cannulated spiral drill was reinserted over the guide wire still in place. The drilling was then completed to a depth $10 \mathrm{~mm}$ less then the subchondral bone as described in the standard operation procedure for DHS ${ }^{\circledR}$ implantation. The subsequent procedure of DHS ${ }^{\circledR}$ implantation was completed without further adaptations. For each patient, the time needed for insertion of the measurement
Fig. 1 a, b Under fluoroscopic control a guide wire is inserted to reach the subchondral bone of the femoral head. A second guide wire is drilled in cranially to prevent rotation of the head neck fragment during subsequent DHS ${ }^{\circledR}$ implantation. The length of the guide wire is measured
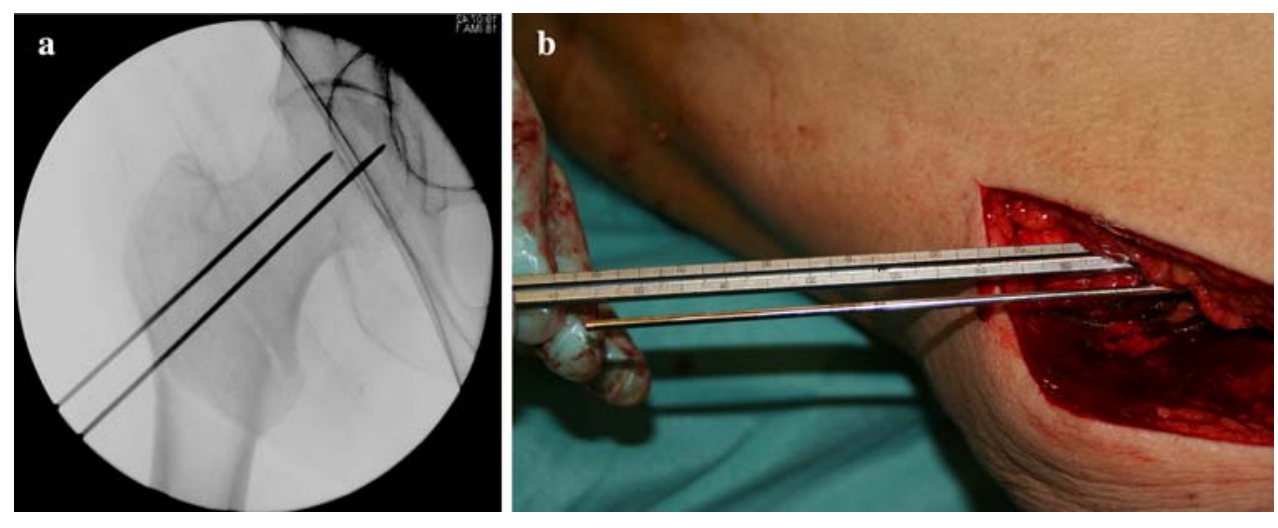

Fig. 2 a, b Reduced pertrochanteric fracture with the guide wire and second cranially placed $\mathrm{k}$-wire to prevent rotation pin in place. The canulated spiral drill is inserted to a depth $45 \mathrm{~mm}$ less than the guide wire
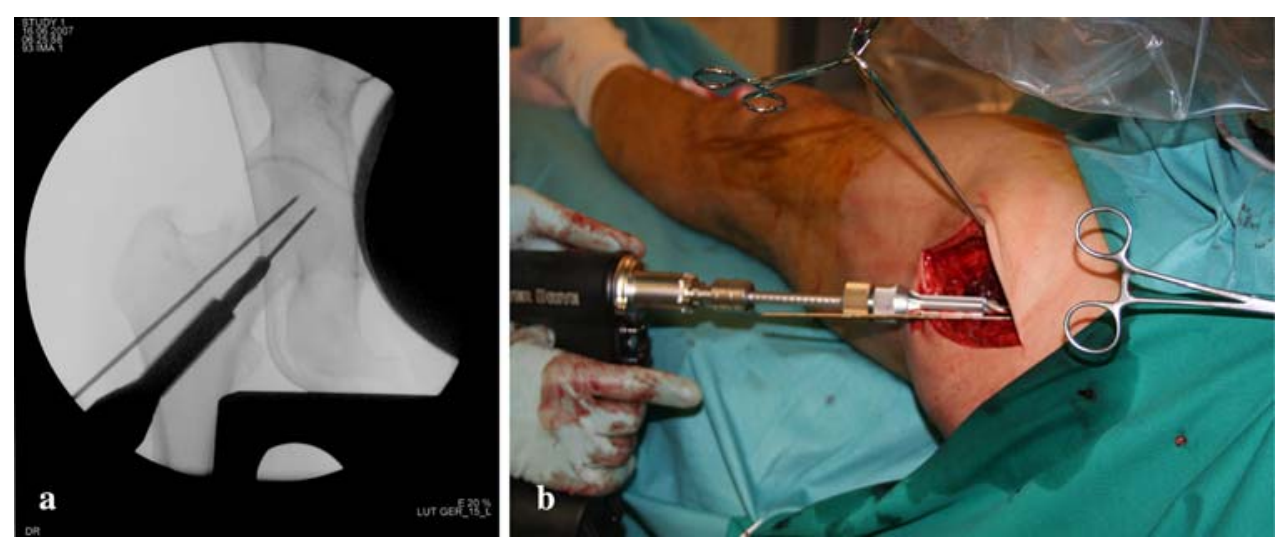


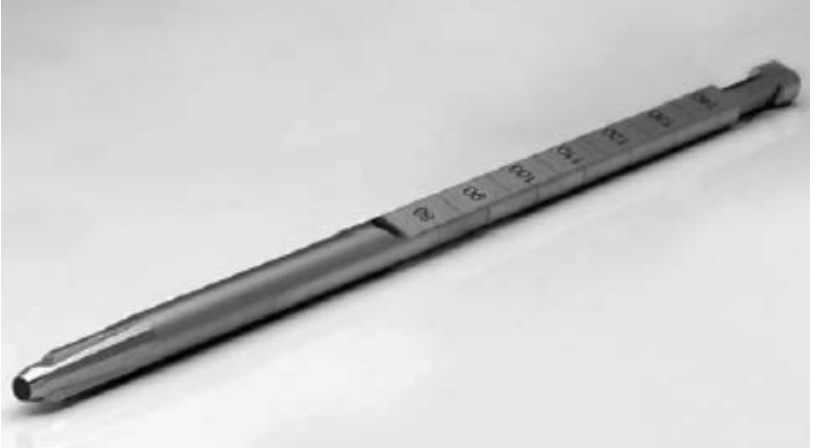

Fig. 3 The cannulated rod like mechanical probe: Central cannulation allows insertion over the guide wire. The tip consists of three blades placed at an angular distance at $120^{\circ}$ each. A measurement scale at the tail allows control of the insertion depth of the probe

blade as well as the mechanical measurement process and the removal of the measurement blade were recorded and related to the total surgery time from skin incision to skin closure.

\section{Complications}

Penetration of the guide wire into the pelvis during insertion of the blade, secondary loss of fracture reduction, wound infection or delayed fracture healing were the worst complications that could be imagined owing to the intraoperative use of DensiProbe ${ }^{\circledR}$. Preventive measures were taken as a result of a risk management process. However, these events may not be prevented in all situations by the measurements taken and they were therefore carefully monitored during the pilot series. Extraction of the guide wire with the blade was also expected, but was looked upon as a minor complication. Moreover, any deviation of the intraoperative procedure from the intended scheme of application described above was documented and analyzed for the potential of a complication.

BMD measurement and postoperative follow-up

Fracture reduction and screw position was recorded on plain radiographs in all patients prior to mobilization under full weight bearing. Patients were then clinically and radiographically followed up at 6 and 12 weeks following surgery for presence of postoperative infection, fracture union, screw loosening or cut out. In the course of postoperative rehabilitation, all patients underwent standard dual energy X-ray absorptiometry (DEXA) to measure BMD of the spine as well as the femoral neck, intertrochanteric and trochanteric area of contralateral unfractured proximal femur. The presence of the implant impaired measurement of the fractured side. But because there is tight correlation between the BMD values of both the proximal femurs,
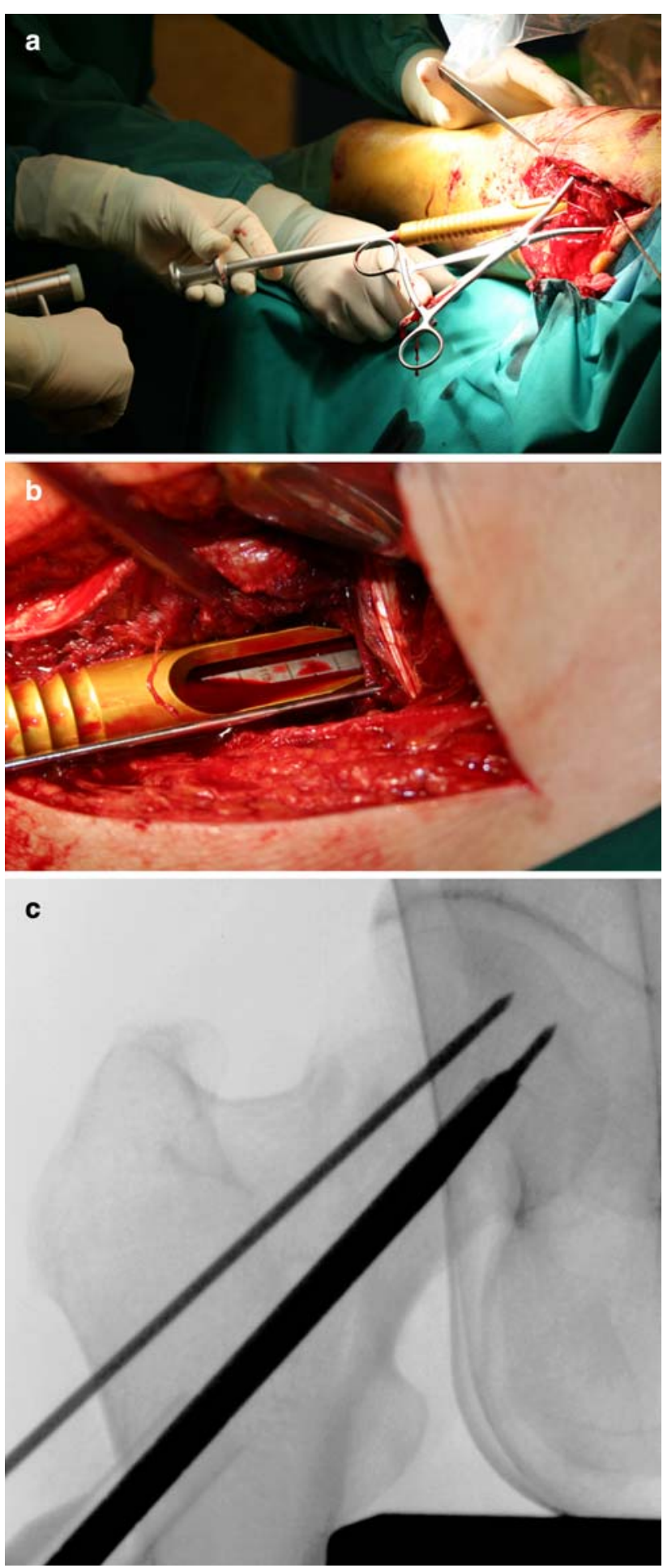

Fig. 4 The cannulated probe is inserted over the guide wire to reach the site of the intended DHS ${ }^{\circledR}$ tip (a). The scale at the tail of the probe ensures control of insertion depth (b). The final position of the probe is documented (c)

hence [22] BMD measurement at the contralateral unfractured side is justified. The BMD values of the femoral neck were then compared to intraoperatively recorded peak torque values. 
Fig. 5 a, b The handhold with its intrinsic digital torque meter is mounted and the probe is rotated along its longitudinal axis. Peak torque to breakaway cancellous bone is registered by the digital torque meter in the handhold
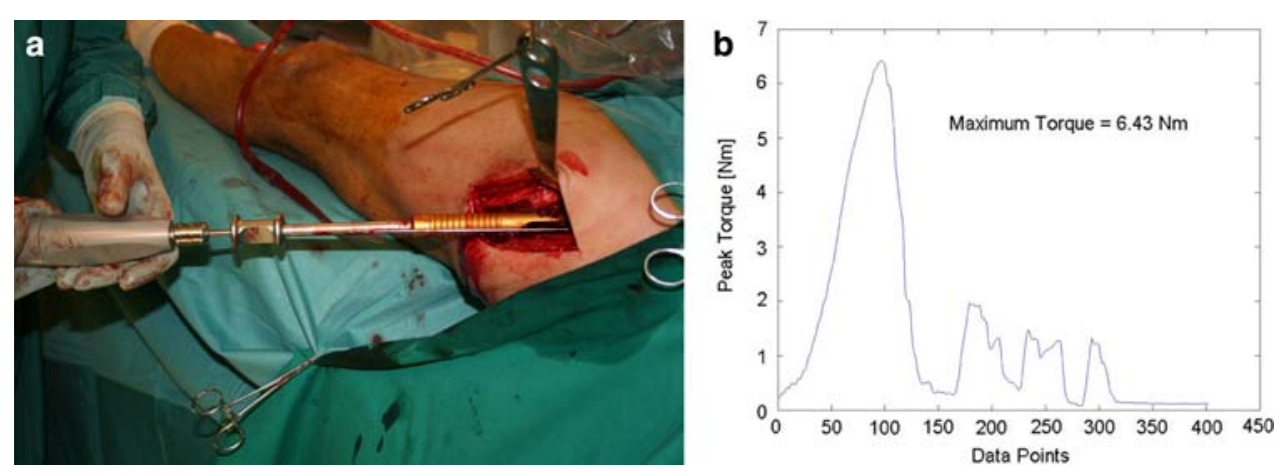

\section{Results}

Patient population

In the inclusion period, 10 patients (six females, four males, mean age: $57 \pm 16$ years) with four pertrochanteric fractures (three, AO31A1; one, AO31A2) and six lateral femoral neck fractures (two Garden 1; three Garden 2; one Garden 4) were enrolled in the study. Six fractures were located on the left side and four fractures on the right side. Table 1 lists all 10 patients with demographic data, type and cause of fracture and presence of BMD modifying conditions.

Seven fractures were caused by a minor trauma, whereas three fractures were the result of an intermediate trauma (fall from a bike, severe direct trauma to the hip during a fight). Four patients reported previous fragility fractures, but no patient indicated familiar burden for such a fracture. Vertebral compression fractures were detected on the chest $\mathrm{X}$-ray in two patients.

Three patients exhibited long-term medication with systemic steroids. Of these, two patients suffered from autoimmune disease (myasthenia gravis and colitis ulcerosa

Table 1 Short characterization of each patient included in the study. The cause of fracture is abbreviated by numbers with 1 corresponding to a low energy trauma such as falling from a standing position and 2 respectively) and one had a history of bone marrow transplantation due to acute myeloic leukemia. Another two patients indicated regular treatment with topical steroids because of asthma. One patient reported regular intake of anticonvulsants in the context of hereditary epilepsia. Three patients were heavy smokers ( $>20 \mathrm{PY}$ ) and another three patients exhibited a BMI lower than $18 \mathrm{~kg} / \mathrm{m}^{2}$. Treatment with calcium/vitamin D had been installed in one patient and no patient was on bisphosphonates.

\section{Peak torque measurement}

No major complication occurred during intraoperative torque measurement. In one case, the guide wire for screw insertion was accidentally pulled out when the cannulated probe was removed from the femoral head. Reinsertion of the guide wire was not problematic. No loss of previously achieved fracture reduction or iatrogenic fracture occurred during the measurement procedures. In the postoperative period, none of the patients showed clinical signs of a surgical site infection. Moreover, fracture healing was uneventful in all the patients. All the fractures united within three months. No screw loosening or cut out was recorded.

corresponding to an intermediate trauma such as falling from a bicycle or a direct blow to the hip

\begin{tabular}{|c|c|c|c|c|}
\hline $\begin{array}{l}\text { Patient } \\
\text { number }\end{array}$ & Age/gender & $\begin{array}{l}\text { Fracture } \\
\text { type }\end{array}$ & $\begin{array}{l}\text { Cause of } \\
\text { fracture }\end{array}$ & BMD modifying conditions \\
\hline 1 & 40/Female & Garden 2 & 1 & Topical steroids (asthma) \\
\hline 2 & 76/Female & AO31A1 & 1 & Vertebral fracture, BMI $15.3 \mathrm{~kg} / \mathrm{m}^{2}$ \\
\hline 3 & 63/Female & Garden 2 & 2 & $\begin{array}{l}\text { Systemic steroids after bone marrow transplantation } \\
\text { due to acute myeloic leucemia, treatment with calcium vitamine } D\end{array}$ \\
\hline 4 & 65/Male & A031A1 & 2 & Systemic steroids due to colitis ulcerosa. \\
\hline 5 & 77/Female & A031A1 & 1 & Vertebral fracture, smoking (77 pack years), BMI $16.9 \mathrm{~kg} / \mathrm{m}^{2}$ \\
\hline 6 & 35/Male & Garden 1 & 2 & Treatment with anticonvulsants \\
\hline 7 & 70/Female & Garden 2 & 1 & None detected \\
\hline 8 & 66/Male & $\mathrm{A} 031 \mathrm{~A} 2$ & 1 & Topical steroids (asthma) \\
\hline 9 & 39/Female & Garden 4 & 1 & Systemic steroids due to myasthenia gravis \\
\hline 10 & 40/Male & Garden 1 & 1 & Smoking (22 pack years) \\
\hline
\end{tabular}




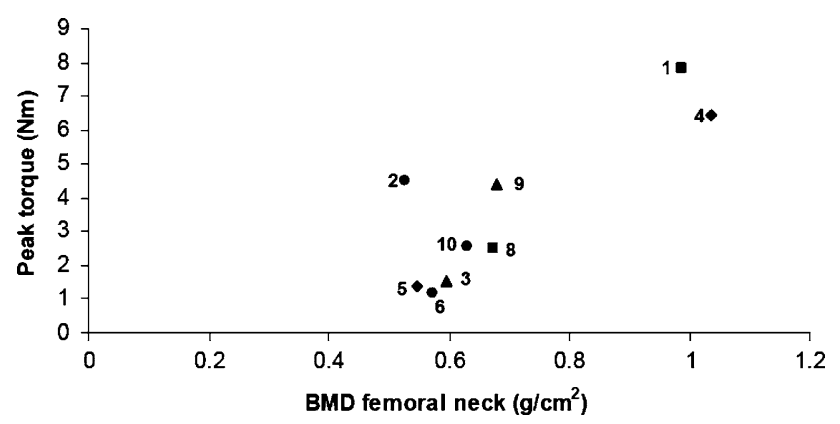

Fig. 6 Intraoperative peak torque values plotted against BMD values measured at the femoral neck

The intraoperative application of the method by trained surgeons never required the presence of any additional person except for data protocol.

The mean time for torque measurement was $2.35 \pm$ 0.9 min accounting for $2.2 \pm 1.1 \%$ of total surgery time. The peak torque as recorded by the digital torque meter ranged from 1.2 to $7.8 \mathrm{Nm}$ with a mean of $3.8 \pm 2.3 \mathrm{Nm}$.

BMD measurement by dual energy X-ray absorptiometry and postoperative follow-up

Postoperative DEXA scans were performed in all but one (patient number seven) who refused investigation of bone mineral density despite previous acceptance with the study protocol. However, this patient received a standard radiographic follow-up.

The mean BMD values of the spine were $0.822 \pm 0.12 \mathrm{~g} /$ $\mathrm{cm}^{2}$. One patient showed normal BMD values of the spine. Osteoporotic $(t$ value $<-2.5)$ and osteopenic spine BMD values ( $t$ value between -1 and -2.5 ) were detected in four patients, respectively. The mean BMD values of the femoral neck were $0.694 \pm 0.187 \mathrm{~g} / \mathrm{cm}^{2}$. In detail, the BMD values at the femoral neck qualified for osteoporosis in four patients, osteopenia in three patients and normal values in two patients. Overall, seven patients were diagnosed with osteoporosis, two with osteopenia and one patient had normal BMD values. The BMD values of the femoral neck of each of the nine patients having undergone DEXA were plotted against intraoperatively measured peak torque values (see Fig. 6). As shown in the graph, there is some tendency towards correlation of peak torque value and BMD.

\section{Discussion}

Despite the limited number of patients included in the pilot study so far, the data presented clearly indicate that the measurement principle and the DensiProbe ${ }^{\circledR}$ instrumentation are eligible for routine intraoperative use by trained surgeons within the framework of a multicenter study. A slight prolongation of the operating time as well as the absence of any relevant complication that could be related to the application of our measurement probe will assure acceptance by the participating surgeons.

Figure 6 presents peak torque values measured with DensiProbe $^{\circledR}$ and BMD values measured by DEXA in the same patients. These data have been included because they outline a number of open questions that might be studied in the future by means of the DensiProbe ${ }^{\circledR}$ method:

- Are we able to identify the cut-off value for peak torque when the application of advanced fixation principles is considered?

- What is the influence of an antiresorptive therapy on bone strength?

The interpretation of possible correlations between these data, however, has to be considered very carefully, because BMD and peak torque analyze bone at two different scales.

The concept of a material's strength is well known from material sciences. For a simple object composed of a uniform material, a break occurs when the load applied creates a stress that exceeds the strength of the material. But bone is a complex composite material with a number of mechanical properties, and no single property adequately describes bone strength [16]. Being aware of this limitation, the strength concept from material sciences might also be applied to bone.

The major noninvasive measurement available for the early diagnosis of osteoporosis is currently the measurement of areal BMD by DEXA. Indeed, BMD is considered a major determinant of bone strength from a macroscopic point of view. Ex vivo studies performed on human material have indicated an excellent correlation between BMD of the proximal femur and bone strength as evaluated by shear test of the femoral neck. BMD does not only integrate the amount of mineral but also indirectly the dimension of bone. BMD predicts approximately $66-74 \%$ of the variation in bone strength. BMD is a surrogate determinant of bone strength to judge the overall status of a skeleton or of a bone as a whole.

On the other hand, the knowledge of bone strength might also help to isolate those patients at risk for loss of reduction, or penetration of a fixation device as complications of open reduction and internal fixation. This information may guide the surgeon in selecting the most appropriate fracture fixation device or technique: This may include hydroxyapatite coated screws or pins which were demonstrated to improve implant osteointegration in various animal [23-26] studies and to enhance fixation strength in osteoporotic bone in randomized controlled clinical trials [27-29]. As an alternative to the use of particular implants designed for osteoporotic bone, the surgeon may consider to augment 
the anchorage of standard implants by the injection of methacrylate bone cement according to our recently published protocol [12]. This easy to apply technique has shown to enhance implant anchorage in osteoporotic cadaver femurs. The associated risk of embolism and clinically relevant tissue necrosis may be lowered by the suggested elution of fat and bone marrow prior to cement application which allows smooth cement distribution in a small volume around the screw tip [4, 30]. Besides these techniques enhancing fixation strength, the surgeon may also decide on a more protective postoperative management. In this context the concept of bone strength is applied to the bone volume right next to the implant.

All terms describing bone strength can be derived from the force-displacement diagram that results from a mechanical test of loading a bone specimen until it breaks [16]. Laboratory studies have therefore been performed where bone became subject of material testing [31, 32]. These test principles can provide good information about bone strength but can only be applied to small samples of bone and are not available in routine clinical use. Nevertheless, these studies form the basis for the idea to perform a simple mechanical test in order to get better information about bone strength.

This idea was first followed in the field of spinal surgery. Several groups have intended to predict pedicle screw loosening or cut out by means of previous intraoperative mechanical analysis of the bone [33-35]. These series have shown uneven results. From our point of view, the pedicle is not an ideal model to apply mechanical measurement of bone strength. Whenever the probe comes into contact with cortical bone, the mechanical competence is completely different from the trabecular bone. This means, the premises to apply a strength concept, e.g., the existence of a homogeneous material is not valid.

We are going to evaluate our measurement principle within the femoral head where we can be pretty sure, the measurement probe does not get into contact with cortical bone. This means, the premise to work with a "strength concept" e.g., the existence of a "homogeneous material" is fulfilled.

Within our stepwise approach to introduce the intraoperative measurement of bone strength into clinical routine, the next step will be a multicenter trial aiming to include several hundreds of patients from different hospitals. Thereby, we may prove that the method can also be applied securely and with reproducible results by such surgeons who were not involved in the development of the concept from the beginning.

Inclusion of sufficient patients to calculate the method's predictive value towards implant cut-out as a single risk factor or in combination with other risk factors can only be achieved by means of a multi center study. Further studies investigating the mechanisms by which drugs reduce fracture risk might also benefit when intraoperative measurement of bone strength by means of DensiProbe ${ }^{\circledR}$ becomes available.

\section{References}

1. Augat P, Rapp S, Claes L (2002) A modified hip screw incorporating injected cement for the fixation of osteoporotic trochanteric fractures. J Orthop Trauma 16(5):311-316

2. Bonnaire F, Zenker H, Lill C, Weber AT, Linke B (2005) Treatment strategies for proximal femur fractures in osteoporotic patients. Osteoporos Int 16(Suppl 2):S93-S102

3. Giannoudis PV, Schneider E (2006) Principles of fixation of osteoporotic fractures. J Bone Joint Surg Br 88(10):1272-1278

4. Gisep A, Curtis R, Flutsch S, Suhm N (2006) Augmentation of osteoporotic bone: effect of pulsed jet-lavage on injection forces, cement distribution, and push-out strength of implants. J Biomed Mater Res B Appl Biomater 78(1):83-88

5. Heini PF (2005) The current treatment-a survey of osteoporotic fracture treatment. Osteoporotic spine fractures: the spine surgeon's perspective. Osteoporos Int 16(Suppl 2):S85-S92

6. Hertel R, Aebi M, Ganz R (1990) Osteosynthesis in high-grade osteoporosis. Unfallchirurg 93(10):479-484

7. Hertel R (2005) Fractures of the proximal humerus in osteoporotic bone. Osteoporos Int 16(Suppl 2):S65-S72

8. Kettunen J, Kroger H (2005) Surgical treatment of ankle and foot fractures in the elderly. Osteoporos Int 16(Suppl 2):S103-S106

9. Kramer A, Angst M, Gasser B, Ganz R (2000) [Increasing bone screw anchoring in the femur head by cement administration via the implant-a biomechanical study]. Z Orthop Ihre Grenzgeb 138(5):464-469

10. Larsson S (2006) Cement augmentation in fracture treatment. Scand J Surg 95(2):111-118

11. Stromsoe K (2004) Fracture fixation problems in osteoporosis. Injury 35(2):107-113

12. von der Linden P, Gisep A, Boner V, Windolf M, Appelt A, Suhm N (2006) Biomechanical evaluation of a new augmentation method for enhanced screw fixation in osteoporotic proximal femoral fractures. J Orthop Res 24(12):2230-2237

13. Parker MJ (1993) Valgus reduction of trochanteric fractures. Injury 24(5):313-316

14. Baumgaertner MR, Curtin SL, Lindskog DM, Keggi JM (1995) The value of the tip-apex distance in predicting failure of fixation of peritrochanteric fractures of the hip. J Bone Joint Surg Am 77(7):1058-1064

15. Baumgaertner MR, Solberg BD (1997) Awareness of tip-apex distance reduces failure of fixation of trochanteric fractures of the hip. J Bone Joint Surg Br 79(6):969-971

16. Felsenberg D, Boonen S (2005) The bone quality framework: determinants of bone strength and their interrelationships, and implications for osteoporosis management. Clin Ther 27(1):1-11

17. Ammann P, Rizzoli R (2003) Bone strength and its determinants. Osteoporos Int 14(Suppl 3):S13-S18

18. Baruffaldi F, Barbanti BG, Testoni M, et al. (1996) Femoral densitometry as potential preoperative indicator for cementation of hip prosthesis. Radiol Med (Torino) 92(3):193-198

19. Suhm N, Hengg C, Schwyn R, Windolf M, Quarz V, Hanni M (2006) Mechanical torque measurement predicts load to implant cut-out: a biomechanical study investigating DHS(R) anchorage in femoral heads. Arch Orthop Trauma Surg 127:469-474

20. Muller ME (1980) [Classification and international AO-documentation of femur fractures]. Unfallheilkunde 83(5):251-259 
21. Garden RS (1974) Reduction and fixation of subcapital fractures of the femur. Orthop Clin North Am 5(4):683-712

22. Yang R, Tsai K, Chieng P, Liu T (1997) Symmetry of bone mineral density at the proximal femur with emphasis on the effect of side dominance. Calcif Tissue Int 61(3):189-191

23. Moroni A, Orienti L, Stea S, Visentin M (1996) Improvement of the bone-pin interface with hydroxyapatite coating: an in vivo long-term experimental study. J Orthop Trauma 10(4):236-242

24. Moroni A, Toksvig-Larsen S, Maltarello MC, Orienti L, Stea S, Giannini S (1998) A comparison of hydroxyapatite-coated, titanium-coated, and uncoated tapered external-fixation pins. An in vivo study in sheep. J Bone Joint Surg Am 80(4):547-554

25. Moroni A, Faldini C, Pegreffi F, Giannini S (2002) Fixation strength of tapered versus bicylindrical hydroxyapatite-coated external fixation pins: an animal study. J Biomed Mater Res 63(1):61-64

26. Moroni A, Faldini C, Giannini S, Wippermann B (2003) Plate fixation with hydroxyapatite-coated screws: a comparative loaded study. Clin Orthop Relat Res 408:262-267

27. Moroni A, Faldini C, Marchetti S, Manca M, Consoli V, Giannini $\mathrm{S}$ (2001) Improvement of the bone-pin interface strength in osteoporotic bone with use of hydroxyapatite-coated tapered externalfixation pins. A prospective, randomized clinical study of wrist fractures. J Bone Joint Surg Am 83-A(5):717-721

28. Moroni A, Faldini C, Pegreffi F, Giannini S (2004) HA-coated screws decrease the incidence of fixation failure in osteoporotic trochanteric fractures. Clin Orthop Relat Res 425:87-92

29. Moroni A, Faldini C, Pegreffi F, Hoang-Kim A, Vannini F, Giannini S (2005) Dynamic hip screw compared with external fixation for treatment of osteoporotic pertrochanteric fractures. A prospective, randomized study. J Bone Joint Surg Am 87(4):753759

30. Gisep A, Curtis R, Hanni M, Suhm N (2006) Augmentation of implant purchase with bone cements: an in vitro study of injectability and dough distribution. J Biomed Mater Res B Appl Biomater 77(1):114-119

31. Krischak GD, Augat P, Wachter NJ, Kinzl L, Claes LE (1999) Predictive value of bone mineral density and Singh index for the in vitro mechanical properties of cancellous bone in the femoral head. Clin Biomech (Bristol, Avon) 14(5):346-351

32. Wachter NJ, Augat P, Hoellen IP et al (2001) Predictive value of Singh index and bone mineral density measured by quantitative computed tomography in determining the local cancellous bone quality of the proximal femur. Clin Biomech (Bristol, Avon) 16(3):257-262

33. Mizuno K, Shinomiya K, Nakai O, Shindo S, Otani K (2005) Intraoperative insertion torque of lumbar pedicle screw and postoperative radiographic evaluation: short-term observation. J Orthop Sci 10(2):137-144

34. Reitman CA, Nguyen L, Fogel GR (2004) Biomechanical evaluation of relationship of screw pullout strength, insertional torque, and bone mineral density in the cervical spine. J Spinal Disord Tech 17(4):306-311

35. Ozawa T, Takahashi K, Yamagata M, et al. (2005) Insertional torque of the lumbar pedicle screw during surgery. J Orthop Sci 10(2):133-136 